\title{
CALAGEM E ADUBAÇÃO ORGÂNICA: INFLUÊNCIA NA ADSORÇÃO DE FÓSFORO EM SOLOS ${ }^{(1)}$
}

\author{
Renato Ferreira de Souza ${ }^{(2)}$, Valdemar Faquin ${ }^{(3)}$, Paulo Rogério \\ Ferreira Torres $^{(4)} \&$ Danielle Pereira Baliza ${ }^{(4)}$
}

\begin{abstract}
RESUMO
A calagem e a adubação orgânica podem reduzir a adsorção/precipitação de $P$ no solo, aumentando sua disponibilidade para a absorção vegetal. Visando avaliar o efeito da calagem e do esterco bovino sobre a adsorção de fósforo no solo, foram realizados em casa de vegetação quatro experimentos no delineamento inteiramente ao acaso, em esquema fatorial $4 \times 5$, com quatro repetições. Os tratamentos foram constituídos por quatro doses de calcário $(0 ; 0,5 ; 1$ e 2 vezes a dose recomendada para atingir $V=60 \%)$ e cinco doses de esterco bovino $(0 ; 2,5 ; 5,0$; 7,5 e $10 \%$ do volume total de solo), aplicados em amostras de $4 \mathrm{dm}^{3} \mathrm{de}$ Neossolo Quartzarênico órtico (RQo), Latossolo Vermelho-Amarelo distrófico textura média (LVAd-1), Latossolo Vermelho-Amarelo distrófico textura argilosa (LVAd-2) e Latossolo Vermelho distrófico textura muito argilosa (LVd), sendo cada solo um experimento. Foram avaliados os teores de fósforo remanescente (P-rem), a capacidade máxima de adsorção de $P$ (CMAP) e o índice tampão de $P$ (ITP) dos solos, os quais foram submetidos ao ajuste de modelos de regressão múltipla, considerando as doses de calcário e de esterco bovino aplicadas. A aplicação de calcário e esterco bovino promoveu a redução da CMAP e o aumento do P-rem e ITP. A alteração nesses valores foi condicionada à mineralogia e à textura, sendo a adsorção de $P$ incrementada com o caráter oxídico dos solos. Com esses resultados, demonstra-se a importância da adoção de sistemas de manejo que contemplem a correção da acidez e elevação dos teores de matéria orgânica dos solos para a otimização do uso do $P$ pelas culturas.
\end{abstract}

Termos de indexação: esterco bovino, calcário, disponibilidade de fósforo, fósforo remanescente, capacidade máxima de adsorção de fósforo, índice tampão de fosfato.

(1) Parte da Tese de Doutorado do primeiro autor, apresentada à Universidade Federal de Lavras - UFLA. Recebido para publicação em setembro de 2005 e aprovado em novembro de 2006.

(2) Assistente de Pesquisa PRODOC - CAPES. Dr. em Solos e Nutrição de Plantas, Universidade Federal de Lavras - UFLA. Rua José Claudino 268, CEP 37200-000 Lavras (MG). E-mail: souzarf2005@yahoo.com.br

(3) Professor do Departamento de Ciência do Solo, UFLA. Bolsista do CNPq.

(4) Graduando em Agronomia, UFLA. 


\title{
SUMMARY: LIMING AND ORGANIC FERTILIZER: INFLUENCE ON PHOSPHORUS ADSORPTION IN SOILS
}

\begin{abstract}
Liming and organic fertilizers can reduce $P$ adsorption/precipitation in the soil, increasing the availability for plant uptake. To evaluate the effect of liming and cattle manure on phosphorus adsorption in the soil, four experiments were conducted in an entirely randomized design, in a $4 \times 5$ factorial layout with four replications, in a greenhouse. The treatments consisted of four liming levels $(0 ; 0,5 ; 1$ and 2 times the recommended dose to reach $V=60 \%)$ and five doses of cattle manure $(0 ; 2,5 ; 5,0 ; 7,5$ and $10 \%$ of the total soil volume), applied in samples of four $d^{3} m^{3}$ of orthic Quartzarenic Neosol sand texture, dystrophic Red-Yellow Latosol sandy clay texture, dystrophic Red-Yellow Latosol sandy clay loam texture and dystrophic Red Latosol clay texture soils; each soil represented an experiment. The values of equilibrium phosphorus (P-rem), maximum $P$ adsorption capacity $(M P A C)$ and the soil $P$ buffering index (PBI) were evaluated and subjected to fitting of multiple regression models as a function of the applied lime and cattle (bovine) manure. Liming and manure fertilizer caused a reduction of MPAC and increase in P-rem and PBI. The alterations of these values was associated to soil mineralogy and texture; $P$ adsorption increased with the oxidic character of the soils. Results evidenced the importance of soil management systems that contemplate acidity correction and increase in organic matter levels to optimize the $P$ use by crops.
\end{abstract}

Index terms: cattle manure, lime, phosphorus availability, equilibrium phosphorus, maximum phosphorus adsorption capacity, phosphate buffer index.

\section{INTRODUÇÃO}

Os solos das regiões tropicais, além da deficiência generalizada, apresentam alta capacidade de fixação de fosfato (adsorção e precipitação), limitando a produtividade das culturas nessas áreas (Raij, 1991). Nesses solos altamente intemperizados, predominam os minerais de argila 1:1, como a caulinita e os óxidos de $\mathrm{Fe}$ (hematita e goethita) e $\mathrm{Al}$ (gibbsita) com alta capacidade de adsorção de P. A magnitude desse fenômeno é influenciada pela natureza e quantidade dos sítios de adsorção, os quais variam de acordo com os fatores intrínsecos e extrínsecos ao próprio solo. Dentre esses fatores, destacam-se a mineralogia, a textura, o pH, o balanço de cargas, a matéria orgânica, o tipo de ácidos orgânicos e a atividade microbiana do solo (Bahia Filho et al., 1983).

O processo de adsorção de $P$ pelos óxidos, hidróxidos e oxiidróxidos de ferro e alumínio é um dos principais fatores envolvidos na insolubilização desse nutriente em solos tropicais (Lopes \& Cox, 1979). A goethita é considerada a principal responsável pelo fenômeno de adsorção de P nos solos do Brasil Central (Bahia Filho et al., 1983). Essa maior capacidade de adsorção de $P$ pelos solos goethíticos foi, também, constatada por Curi \& Franzmeier (1984), sendo creditada à facilidade de acesso do fosfato aos grupos $\mathrm{OH}^{-}$de superfície (Frossard et al., 1994). Ocorrem, também, reações de adsorção de fosfato por minerais de argila que apresentam grupos $\mathrm{OH}$ expostos, tal como a caulinita, que apresenta alta afinidade pelo $\mathrm{P}$ (Mcbride, 1994). De acordo com esse autor, em condições de solo ácido, o ín fosfato reage rapidamente com o octaedro de $\mathrm{Al}$, pela substituição dos grupos $\mathrm{OH}$ localizados na superfície do mineral, formando complexos de esfera interna. Ocorrem, também, reações de precipitação do $\mathrm{P}$ com formas iônicas de $\mathrm{Al}$ e $\mathrm{Fe}$ em solos ácidos e Ca em meio básico, formando compostos de baixa solubilidade (Novais \& Smyth, 1999).

A adoção de sistemas de manejo do solo, visando ao aumento de matéria orgânica, aumento do $\mathrm{pH}$ e maior atividade da microbiota do solo, pode interferir no processo, reduzindo a adsorção de P. Os grupos funcionais carboxílicos e fenólicos presentes na matéria orgânica são responsáveis pelo bloqueio dos sítios de carga positiva dos óxidos de $\mathrm{Fe}$ e $\mathrm{Al}$, reduzindo a adsorção do $\mathrm{P}$ (Hue, 1991). A elevação do $\mathrm{pH}$ do solo pela calagem, aumentando a concentração e atividade dos íons $\mathrm{OH}^{-}$em solução, promove a precipitação de $\mathrm{Fe}$ e $\mathrm{Al}$, reduzindo a precipitação de $\mathrm{P}-\mathrm{Fe}$ e $\mathrm{P}-\mathrm{Al}$ de baixa solubilidade; há, também, geração de cargas negativas pela desprotonação de hidroxilas expostas nas argilas e matéria orgânica, ocorrendo repulsão entre o fosfato e a superfície adsorvente (Mcbride, 1994). Assim, o manejo a que o solo é submetido, alterando os fatores envolvidos no processo, pode reduzir a adsorção de $\mathrm{P}$ no solo, aumentando a sua disponibilidade às plantas.

Objetivou-se, com esse trabalho, avaliar o efeito da adição de esterco bovino e de calcário sobre a adsorção de fósforo, em quatro solos com diferentes texturas, mineralogia e teor de matéria orgânica. 


\section{MATERIAL E MÉTODOS}

Quatro experimentos foram realizados no Departamento de Ciência do Solo da Universidade Federal de Lavras, com amostras de quatro solos bem drenados, predominantes no Estado de Minas Gerais, e com ampla variação nos teores de argila, mineralogia e matéria orgânica (MO), constituindo cada solo um experimento especifico. Para abranger ampla variação nas características físicas, químicas e mineralógicas, foram utilizados os solos classificados no sistema brasileiro de classificação de solos (Embrapa, 1999) como: Neossolo Quartzarênico órtico fase cerrado (RQo), Latossolo Vermelho-Amarelo distrófico textura média fase floresta tropical subperenifólia (LVAd-1), Latossolo Vermelho-Amarelo distrófico textura argilosa fase floresta tropical subperenifólia (LVAd-2) e Latossolo Vermelho distrófico textura muito argilosa fase floresta tropical subperenifólia (LVd). O solo RQo foi coletado no município de Itutinga-MG (coordenadas UTM: x: 0540201 e y: 7644073 - 23K), o LVAd-1 foi proveniente do município de Itumirim-MG (coordenadas UTM: x: 0521106 e y: 7646097 - 23K), o LVAd-2 (coordenadas UTM: x: 0504202 e y: 7652821 -
23K) e o LVd (coordenadas UTM: x: 0503646 e y: 7652507 - 23K) foram coletados no Campus da Universidade Federal de Lavras, Lavras-MG.

As amostras dos solos foram coletadas na profundidade de $0-20 \mathrm{~cm}$, sob vegetação natural, após retirar a vegetação e os restos orgânicos presentes na superfície. Após a coleta, as amostras foram caracterizadas física, química e mineralogicamente (Quadro 1). A granulometria dos solos foi determinada pelo método da pipeta (Day, 1965), empregando-se $\mathrm{NaOH} 0,1 \mathrm{~mol} \mathrm{~L}^{-1}$ como dispersante químico e agitação rápida, sendo a fração areia $(2-0,053 \mathrm{~mm})$ separada por meio de tamisagem. $\mathrm{O} \mathrm{pH}$ em água, $\mathrm{Ca}, \mathrm{Mg}, \mathrm{Al}, \mathrm{P}, \mathrm{K}$ foram determinados conforme EMBRAPA (1997), sendo o Ca, Mg e Al extraídos com $\mathrm{KCl} 1 \mathrm{~mol} \mathrm{~L}^{-1}$ e o P e K pelo $\mathrm{HCl}$ 0,05 mol L-1 $+\mathrm{H}_{2} \mathrm{SO}_{4}$ $0,0125 \mathrm{~mol} \mathrm{~L}^{-1}$ (Mehlich-1). Também foram determinados a acidez potencial $(\mathrm{H}+\mathrm{Al})$ e o $\mathrm{C}$ orgânico, conforme Raij et al. (1987). O fósforo remanescente foi determinado conforme, Alvarez V. et al. (2000). Na fração argila, foram quantificadas caulinita (Ct) e gibbsita (Gb), mediante Análise TermoDiferencial (ATD), sendo as amostras da mesma fração submetidas à difração de raios-X (método do pó) (Klug

Quadro 1. Principais atributos químicos, físicos e mineralógicos das amostras dos solos, coletadas sob condições naturais, na camada de $0-20 \mathrm{~cm}$

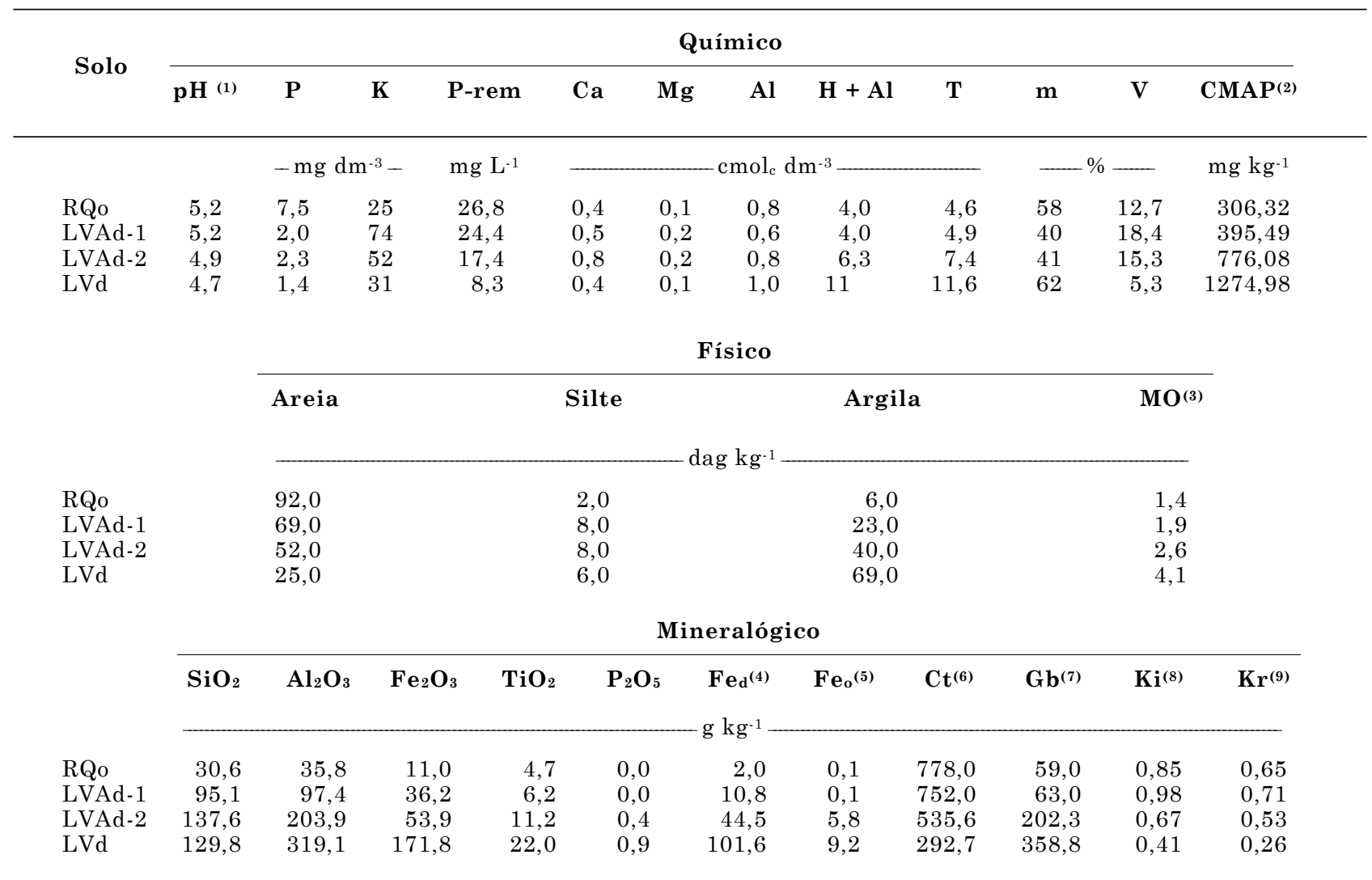

\footnotetext{
(1) $\mathrm{pH}$ em água. ${ }^{(2)}$ Capacidade máxima de adsorção de $\mathrm{P} .{ }^{(3)} \mathrm{MO}=\% \mathrm{C} \times 1,723 .{ }^{(4)}$ Óxidos de Fe livre totais. ${ }^{(5)}$ Óxidos de Fe menos cristalinos. ${ }^{(6)}$ Caulinita. ${ }^{(7)}$ Gibbsita. ${ }^{(8)} \mathrm{Ki}=\mathrm{SiO}_{2} / \mathrm{Al}_{2} \mathrm{O}_{3} .{ }^{(9)} \mathrm{Kr}=\mathrm{SiO}_{2} / \mathrm{Al}_{2} \mathrm{O}_{3}+\mathrm{Fe}_{2} \mathrm{O}_{3}$.
} 
\& Alexander, 1974), óxidos de ferro livre totais $\left(\mathrm{Fe}_{\mathrm{d}}\right)$, usando o ditionito-citrato-bicarbonato de sódio (DCB) (Mehra \& Jackson, 1960) e óxidos de ferro menos cristalinos $\left(\mathrm{Fe}_{\mathrm{o}}\right)$ extraídos segundo método de Schwertmann (1964), usando-se oxalato ácido de amônio e, em ambos os casos, o Fe foi determinado por espectrofotometria de absorção atômica (Krishna Murti et al., 1966). Os óxidos $\left(\mathrm{SiO}_{2}, \mathrm{Al}_{2} \mathrm{O}_{3}, \mathrm{Fe}_{2} \mathrm{O}_{3}\right.$, $\mathrm{TiO}_{2}$ e $\mathrm{P}_{2} \mathrm{O}_{5}$ ) foram determinados pelo ataque sulfúrico, segundo Embrapa (1997), através dos quais foram determinados $\mathrm{Ki}=\mathrm{SiO}_{2} / \mathrm{Al}_{2} \mathrm{O}_{3}$ e $\mathrm{Kr}=\mathrm{SiO}_{2} /$ $\mathrm{Al}_{2} \mathrm{O}_{3}+\mathrm{Fe}_{2} \mathrm{O}_{3}$.

Os experimentos foram realizados em casa de vegetação, utilizando-se o delineamento experimental inteiramente casualizado (DIC), em esquema fatorial $4 \times 5$, com quatro repetições, sendo cada unidade experimental constituída por um vaso com capacidade de $4 \mathrm{dm}^{3}$ de solo. Os tratamentos foram constituídos de quatro doses de calcário, equivalentes a $0 ; 0,5 ; 1,0$ e 2,0 vezes as doses recomendadas para elevar a saturação por bases em cada solo a $60 \%$ e cinco doses de esterco bovino decomposto $(0,100,200,300$ e $400 \mathrm{~cm}^{3}$ ), correspondendo a $0 ; 2,5 ; 5,0 ; 7,5$ e $10 \%$ do volume de solo de cada unidade experimental.

Para sua incorporação aos solos, o esterco bovino foi seco a $60^{\circ} \mathrm{C}$ e moído em moinho com malha de $1 \mathrm{~mm}^{2}$, apresentando densidade de $462 \mathrm{~g} \mathrm{dm}^{-3}$; a análise química do esterco apresentou os seguintes teores de nutrientes: 18,$0 ; 3,4 ; 14,2 ; 12,1 ; 6,0$ e $2,6 \mathrm{~g} \mathrm{~kg}^{-1}$ de N, P, K, Ca, Mg e S e 21,9; 11012,0; 449,0 e 78,5 mg kg-1 de $\mathrm{Cu}, \mathrm{Fe}, \mathrm{Mn}$ e $\mathrm{Zn}$, respectivamente. Utilizou-se um calcário dolomítico calcinado e micropulverizado, com $35 \%$ de $\mathrm{CaO}, 14 \%$ de $\mathrm{MgO}$ e PRNT de $100 \%$.

Após um período de 30 dias de incubação, com a umidade dos solos mantida próxima à capacidade de campo, determinaram-se os valores de fósforo remanescente (P-rem), da capacidade máxima de adsorção de fósforo (CMAP) e de dessorção de $\mathrm{P}$, a partir dos quais se estimou o índice tampão de fosfato (ITP).

O P-rem foi determinado na solução de equilíbrio solo - solução, obtida pela agitação de $5 \mathrm{~cm}^{3}$ de TFSA com $50 \mathrm{~mL}$ de uma solução de $\mathrm{CaCl}_{2} 10 \mathrm{mmol} \mathrm{L}^{-1}$ que continha $60 \mathrm{mg} \mathrm{L}^{-1}$ de P, por uma hora (Alvarez V. et al., 2000). Após centrifugar e filtrar, o P na solução de equilíbrio foi dosado pelo método da vitamina $\mathrm{C}$, modificado por Braga \& Defelipo (1974).

Para a determinação da CMAP (Olsen \& Watanabe, 1957), os solos foram separados de acordo com o P-rem, enquanto as doses de P para cada solo foram estabelecidas, segundo Alvarez V. \& Fonseca (1990), para a confecção das isotermas de adsorção $(0,0 ; 0,1 ; 0,25 ; 0,45 ; 0,70$ e 1,0 vez a concentração máxima de $\mathrm{P}$ em cada solo). Na determinação do $\mathrm{P}$ adsorvido, $2,5 \mathrm{~cm}^{3}$ de TFSA foram agitados, por $24 \mathrm{~h}$, com $25 \mathrm{~mL}$ de uma solução de $\mathrm{CaCl}_{2} 10 \mathrm{mmol} \mathrm{L}^{-1}$ que continha P nas concentrações estabelecidas para cada solo (RQo e LVAd-1: 0 a $80 \mathrm{mg} \mathrm{L}^{-1}$; LVAd-2: 0 a $110 \mathrm{mg} \mathrm{L}^{-1} \mathrm{e} \mathrm{LVd:} 0$ a $150 \mathrm{mg} \mathrm{L}^{-1}$ ). Após centrifugação e filtragem, o P da solução de equilíbrio foi dosado colorimetricamente (Braga \& Defelipo, 1974). A diferença entre a quantidade inicial de $\mathrm{P}$ na solução e a quantidade na solução de equilíbrio foi considerada como sendo o $\mathrm{P}$ adsorvido. Os valores de $\mathrm{P}$ adsorvido foram ajustados à equação de Langmuir para estimar a CMAP, de acordo com a equação:

$$
x / m=k b C /(1+k C)
$$

em que $\mathrm{x} / \mathrm{m}=$ fósforo adsorvido ao solo $[\mathrm{mg} \mathrm{P}(\mathrm{x}) / \mathrm{g}$ solo $(m)] ; k=$ constante relacionada com a energia de ligação do $\mathrm{P}\left(\mathrm{L} \mathrm{mg}^{-1}\right) ; b=\mathrm{CMAP}$ do solo $\left(\mathrm{mg} \mathrm{g}^{-1}\right)$, e $C$ $=$ concentração de $\mathrm{P}$ na solução de equilíbrio $\left(\mathrm{mg} \mathrm{L}^{-1}\right)$.

Para a obtenção das estimativas das constantes $k$ $e b$, utilizou-se a forma linearizada da equação de Langmuir:

$$
C / x / m=1 / k b+C / b
$$

Nas mesmas amostras de solo, após a centrifugação e retirada do sobrenadante, foram adicionados $30 \mathrm{~mL}$ de $\mathrm{CaCl}_{2} 10 \mathrm{mmol} \mathrm{L}^{-1} \mathrm{e}$ agitados por $24 \mathrm{~h}$. Após esse período, a mistura solo/solução foi novamente centrifugada e filtrada e o $\mathrm{P}$ do sobrenadante dosado por colorimetria, para determinação do $\mathrm{P}$ dessorvido. Equações de regressão quadrática foram ajustadas entre o $\mathrm{P}$ adsorvido e o $\mathrm{P}$ dessorvido nas diferentes concentrações de $\mathrm{P}$ adicionadas para cada tratamento. A partir do inverso dos coeficientes " $b$ " (1/b) das equações (declividade da curva de regressão), foram estimados índices tampão de fosfato (ITP), adaptados de Accioly et al. (1985) e Guilherme et al. (2000). O ITP representa a resistência do solo a mudanças do $\mathrm{P}$ lábil como conseqüência de alterações no $\mathrm{P}$ da solução. Quanto maiores os valores de ITP, menor a resistência de liberação de $\mathrm{P}$ da fração lábil para a solução do solo, ou seja, menor o fator capacidade de P (FCP) no solo (ITP = 1/FCP) (Fox \& Kamprath, 1970).

Passado o período de incubação, a cada unidade experimental (vasos com $3,5 \mathrm{dm}^{3}$ de solo), foram aplicadas as doses de 200, 250, 320 e $400 \mathrm{mg} \mathrm{dm}^{-3} \mathrm{de}$ $\mathrm{P}$, para os solos RQo, LVAd-1, LVAd-2 e LVd, respectivamente, de acordo com Alvarez V. \& Fonseca (1990), e uma adubação básica com macro e micronutrientes, utilizando-se como fontes reagentes p.a. Após incubação por mais 30 dias, cultivaram-se duas plantas de feijoeiro (Phaseolus vulgaris L., vc. Carioca MG) até o final do ciclo, avaliando-se a produção de matéria seca de grãos e o acúmulo de $\mathrm{P}$ na parte aérea total (parte aérea + grãos), cujos resultados estão em Souza et al. (2006).

Os valores das variáveis P-rem, CMAP e ITP foram submetidos ao ajuste de modelos de regressão múltipla (superfícies de resposta), considerando as doses de calcário e de esterco bovino aplicadas (Alvarez V., 1994). Os modelos ajustados foram selecionados conforme o procedimento stepwise, isto é, considerando-se um 
modelo geral completo, selecionou-se um modelo menor (submodelo), nos quais todos os parâmetros foram significativos (Khuri \& Cornell, 1996). Foi utilizado o programa de análises estatísticas $\mathrm{R}(\mathrm{R}$ Development Core Team, 2004). Para os solos RQo, LVAd-1 e LVAd-2, foi necessária a transformação dos dados de índice tampão de fósforo (ITP), para que os erros dos modelos ajustados atendessem às pressuposições de normalidade e homogeneidade de variâncias. As transformações foram:

$$
\operatorname{ITPt}(R Q)=\sqrt{1 / I_{T P}^{+0,5}} \text { e } \operatorname{ITPt}(\operatorname{LVAd} 1 e 2)=\log \left[\frac{1}{\operatorname{ITP}}\right]
$$

Os valores do P-rem, CMAP e ITP foram correlacionados com os dados de produção de grãos e acúmulo de $\mathrm{P}$ na parte aérea do feijoeiro, apresentados por Souza et al. (2006).

\section{RESULTADOS E DISCUSSÃO}

A adição de esterco bovino e de calcário proporcionou aumentos significativos dos valores de P-rem e redução nos valores da CMAP (Figura 1 e Quadros 2 e 3). Esse comportamento pode ser atribuído ao bloqueio dos sítios de adsorção de P no solo pela matéria orgânica (MO) adicionada, onde os grupos funcionais carboxílicos e fenólicos dos ácidos orgânicos se ligam às hidroxilas dos óxidos de $\mathrm{Fe}$ e $\mathrm{Al}$ e complexam o $\mathrm{Al}$ em solução (Hue, 1991). Segundo Haynes (1984), o solo pode adsorver ácidos orgânicos com grande energia, competindo com sítios de adsorção de $\mathrm{P}$ e aumentando a disponibilidade desse nutriente para as plantas. A matéria orgânica pode originar, também, ligantes orgânicos liberados durante o processo de mineralização, que podem formar complexos com $\mathrm{Al}$ ou complexos solúveis com o P da solução do solo, evitando que o mesmo seja adsorvido (Iyamuremye et al., 1996). Todavia, com as maiores doses de esterco bovino, verificou-se tendência de redução nos valores de P-rem e aumento na CMAP (Figura 1). Isso deveuse, possivelmente, à adsorção de $\mathrm{P}$ aos compostos húmicos, uma vez que o caráter aniônico da MO, por via de pontes de cátions ( $\mathrm{Al}, \mathrm{Fe}$ e Ca), retém o $\mathrm{P}$ (Novais \& Smyth, 1999).

$\mathrm{O} \mathrm{Al}$ e, ou, Fe em solução podem causar precipitação de fosfatos adicionados ao solo. A calagem, elevando o pH do solo pela produção de hidroxilas, promove a precipitação de $\mathrm{Fe}$ e de $\mathrm{Al}$ trocáveis, reduzindo a precipitação de $\mathrm{P}$ com esses dois metais. Entretanto, a retenção de $\mathrm{P}$ pelo solo mantém valores estequiométricos semelhantes, quando se substitui a precipitação do $\mathrm{P}$ pelo $\mathrm{Al}^{3+}$ do solo ácido pela sua adsorção pelo hidróxido de $\mathrm{Al}$ recém-formado pela calagem (Haynes, 1984). A elevação do $\mathrm{pH}$ promove, também, a desprotonação de hidroxilas de radicais orgânicos e daquelas expostas na superfície das argilas, aumentando a repulsão (menor adsorção) entre o fosfato e a superfície adsorvente e diminuindo o potencial eletrostático (McBride, 1994; Novais \& Smyth, 1999). Por outro lado, com o aumento do $\mathrm{pH}$, ocorre a diminuição da forma $\mathrm{H}_{2} \mathrm{PO}_{4}^{-}$, em relação à $\mathrm{HPO}_{4}^{2-}$, esta (bivalente) preferencialmente adsorvida, contrabalançando o decréscimo do potencial eletrostático (Novais \& Smyth, 1999). Também, segundo esses autores, com uma elevada dose de calcário e maior teor de Ca trocável, pode ocorrer a precipitação de $\mathrm{P}$, formando fosfatos bi e tricálcicos de baixa solubilidade, explicando a redução nos valores de P-rem e aumento na CMAP com as doses mais altas de calcário.

A calagem de solos ricos em $\mathrm{Al}^{3+}$ tende a aumentar a adsorção de $\mathrm{P}$, diminuindo-a em solos pobres em $\mathrm{Al}^{3+}$, pois polímeros de hidróxidos de $\mathrm{Al}$ recém formados com a calagem apresentam alta afinidade pelo $\mathrm{P}$ (Haynes, 1984). De acordo com esses autores, os polímeros catiônicos de $\mathrm{Al}$ formados com a calagem podem, também, capear colóides do solo, restringindolhes a carga negativa. De acordo com Novais \& Smyth, (1999), considerando que, dentre as formas minerais (P-Al, $\mathrm{P}-\mathrm{Fe}$ e $\mathrm{P}-\mathrm{Ca}$ ), o $\mathrm{P}-\mathrm{Al}$ é a que melhor representa o P-lábil do solo, essa retenção não deve ser a pior opção quanto à disponibilidade de $\mathrm{P}$ às plantas.

Embora os solos não tenham sido comparados estatisticamente, os valores absolutos da CMAP, obtidos a partir das isotermas de Langmuir, obedeceram à seguinte ordem decrescente: LVd > LVAd-2 > LVAd-1 > RQo, ocorrendo o inverso com os valores de P-rem (Figura 1 e Quadros 2 e 3). Nesta ordem, os solos apresentam teores decrescentes de MO, $\mathrm{Fe}_{\mathrm{d}}, \mathrm{Fe}_{\mathrm{o}}$, gibbsita, argila e crescentes de $\mathrm{Ki}$ e $\mathrm{Kr}$ (Quadro 1). À medida que os solos apresentam maior teor de óxidos, aumenta a adsorção de $\mathrm{P}$ bem como seu conteúdo nas formas ligadas a $\mathrm{Al}$ e Fe. A adsorção de $\mathrm{P}$ por óxidos é apenas parcialmente reversível, ocorrendo o fenômeno de histerese, ou seja, a quantidade de $\mathrm{P}$ dessorvida é inferior à quantidade adsorvida. A goethita tem sido considerada como o principal componente da fração argila responsável pela adsorção de P em solos do Brasil Central (Bahia Filho et al., 1983). A influência da substituição isomórfica de Fe por $\mathrm{Al}$ e da maior superfície específica da goethita sobre o fenômeno de adsorção de $\mathrm{P}$ também tem sido citada na literatura (Resende, 1976), bem como o efeito da morfologia dos cristais (Torrent et al., 1990; Ruan \& Gilkes, 1996). A gibbsita tem participação importante na adsorção de $\mathrm{P}$, embora de forma menos efetiva que a goethita. Porém, em razão da sua ocorrência em grandes quantidades em alguns Latossolos Argilosos brasileiros, sua contribuição, em termos de adsorção total, pode ultrapassar a dos óxidos de ferro (Curi et al., 1988). Os solos que apresentaram maiores valores de CMAP e menores de P-rem corresponderam àqueles com menores valores de $\mathrm{Ki}\left(\mathrm{SiO}_{2} / \mathrm{Al}_{2} \mathrm{O}_{3}\right)$ e $\mathrm{Kr}\left(\mathrm{SiO}_{2} /\right.$ $\mathrm{Al}_{2} \mathrm{O}_{3}+\mathrm{Fe}_{2} \mathrm{O}_{3}$ ) (Quadro 1), concordando com resultados obtidos por Curi et al. (1988). 

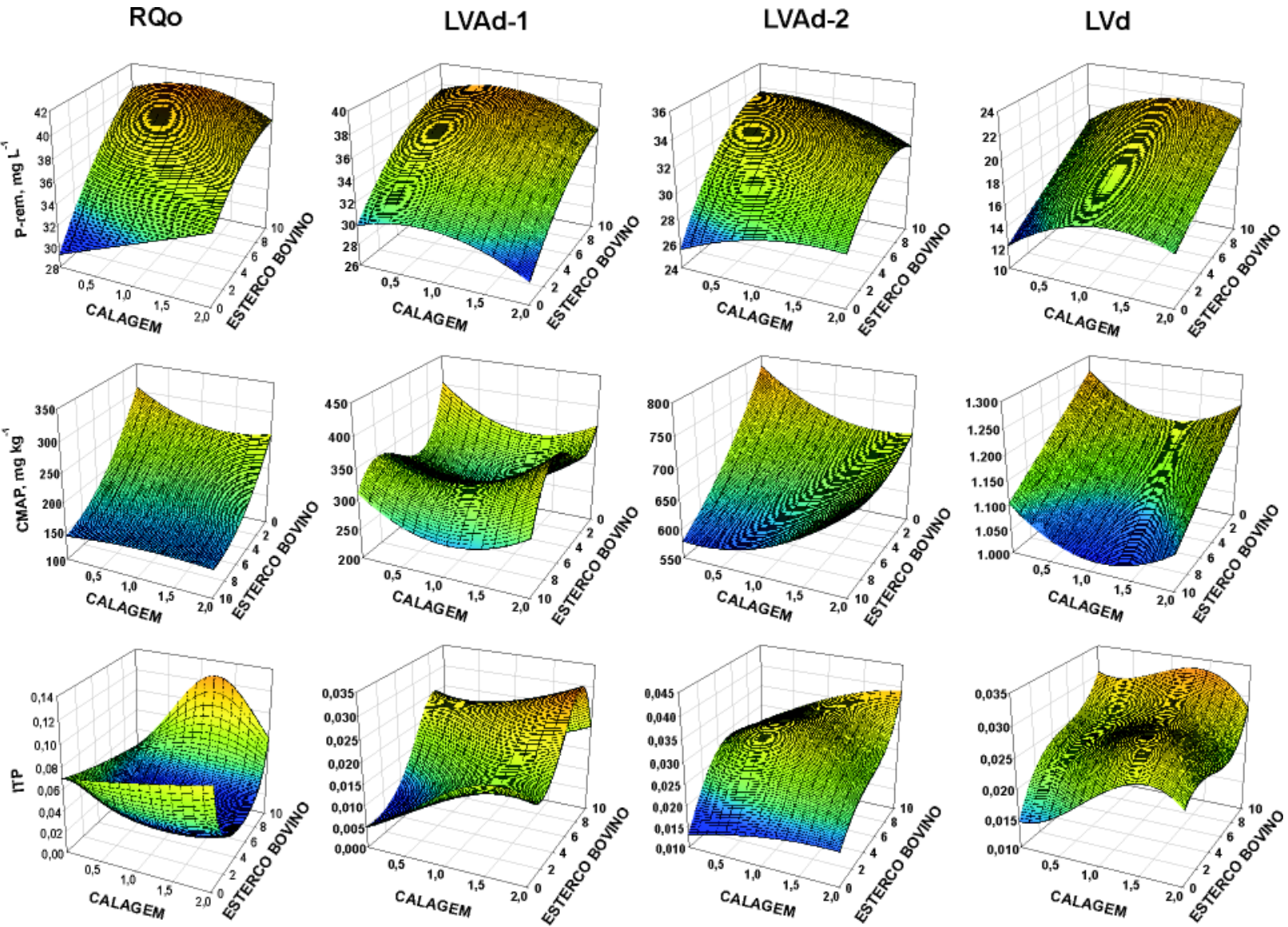

Figura 1. Fósforo remanescente (P-rem), capacidade máxima de adsorção de P (CMAP) e índice tampão de fosfato (ITP), considerando a aplicação de esterco bovino (\% do volume de solo) e calagem (vezes a dose para elevar $\mathrm{V}=60 \%$ ).

Quadro 2. Modelos ajustados com os resultados de fósforo remanescente (P-rem), capacidade máxima de adsorção de fósforo (CMAP) e do índice tampão de fosfato (ITP) considerando a aplicação de esterco bovino (MO) e calcário (CAL), nos solos RQo, LVAd-1, LVAd-2 e LVd

\begin{tabular}{|c|c|c|}
\hline Solo & Modelo ajustado & $\mathbf{R}^{2}$ \\
\hline RQo & $\mathrm{P}-\mathrm{rem}=\beta_{0}+\beta_{1} \mathrm{MO}+\beta_{2} \mathrm{CAL}+\beta_{3} \mathrm{MO}^{2}+\beta_{4} \mathrm{MO} * \mathrm{CAL}^{2}$ & 0,87 \\
\hline LVAd-1 & $\mathrm{P}-$ rem $=\beta_{0}+\beta_{1} \mathrm{MO}+\beta_{2} \mathrm{CAL}+\beta_{3} \mathrm{MO}^{2}+\beta_{4} \mathrm{CAL}^{2}$ & 0,84 \\
\hline LVAd-2 & $\mathrm{P}-\mathrm{rem}=\beta_{0}+\beta_{1} \mathrm{MO}+\beta_{2} \mathrm{CAL}+\beta_{3} \mathrm{MO}^{2}+\beta_{4} \mathrm{CAL}^{2}+\beta_{5} \mathrm{MO} * \mathrm{CAL}$ & 0,84 \\
\hline LVd & $\mathrm{P}-\mathrm{rem}=\beta_{0}+\beta_{1} \mathrm{MO}+\beta_{2} \mathrm{CAL}+\beta_{3} \mathrm{CAL}^{2}+\beta_{4} \mathrm{CAL} * \mathrm{MO}^{2}$ & 0,83 \\
\hline RQo & $\mathrm{CMAP}=\beta_{0}+\beta_{1} \mathrm{MO}+\beta_{2} \mathrm{CAL}+\beta_{3} \mathrm{MO}^{2}+\beta_{4} \mathrm{CAL}^{2}+\beta_{5} \mathrm{MO} * \mathrm{CAL}+\beta_{6} \mathrm{MO} * \mathrm{CAL}^{2}$ & 0,90 \\
\hline LVAd-1 & $\mathrm{CMAP}=\beta_{0}+\beta_{1} \mathrm{MO}+\beta_{2} \mathrm{CAL}+\beta_{3} \mathrm{MO}^{2}+\beta_{4} \mathrm{CAL}^{2}+\beta_{5} \mathrm{MO}^{3}+\beta_{6} \mathrm{MO} * \mathrm{CAL}+\beta_{7} \mathrm{MO}^{2} * \mathrm{CAL}$ & 0,70 \\
\hline LVAd-2 & $\mathrm{CMAP}=\beta_{0}+\beta_{1} \mathrm{MO}+\beta_{2} \mathrm{CAL}+\beta_{3} \mathrm{MO}^{2}+\beta_{4} \mathrm{CAL}^{2}+\beta_{5} \mathrm{MO} * \mathrm{CAL}$ & 0,68 \\
\hline LVd & $\mathrm{CMAP}=\beta_{0}+\beta_{1} \mathrm{MO}+\beta_{2} \mathrm{CAL}+\beta_{3} \mathrm{CAL}^{2}$ & 0,64 \\
\hline RQo & $\mathrm{ITP}_{\mathrm{t}}=\beta_{0}+\beta_{1} \mathrm{MO}+\beta_{2} \mathrm{CAL}+\beta_{3} \mathrm{MO}^{2}+\beta_{4} \mathrm{CAL}^{2}+\beta_{5} \mathrm{MO} * \mathrm{CAL}+\beta_{6} \mathrm{MO} * \mathrm{CAL}^{2}$ & 0,80 \\
\hline LVAd-1 & $\mathrm{ITP}_{\mathrm{t}}=\beta_{0}+\beta_{1} \mathrm{MO}+\beta_{2} \mathrm{CAL}+\beta_{3} \mathrm{MO}^{2}+\beta_{4} \mathrm{CAL}^{2}+\beta_{5} \mathrm{MO}^{3}+\beta_{6} \mathrm{MO} * \mathrm{CAL}+\beta_{7} \mathrm{MO} * \mathrm{CAL}^{2}$ & 0,63 \\
\hline LVAd-2 & $\mathrm{ITP}_{\mathrm{t}}=\beta_{0}+\beta_{1} \mathrm{MO}+\beta_{2} \mathrm{CAL}+\beta_{3} \mathrm{MO}^{2}+\beta_{4} \mathrm{CAL}^{2}+\beta_{5} \mathrm{MO}^{3}+\beta_{6} \mathrm{MO} * \mathrm{CAL}^{2}+\beta_{7} \mathrm{MO}^{2} * \mathrm{CAL}^{2}$ & 0,87 \\
\hline LVd & ITP $=\beta_{0}+\beta_{1} \mathrm{MO}+\beta_{2} \mathrm{CAL}+\beta_{3} \mathrm{MO}^{2}+\beta_{4} \mathrm{CAL}^{2}+\beta_{5} \mathrm{MO}^{3}+\beta_{6} \mathrm{CAL}^{3}+\beta_{7} \mathrm{MO} * \mathrm{CAL}+\beta_{8} \mathrm{CAL}^{*} \mathrm{MO}^{2}$ & 0,76 \\
\hline
\end{tabular}


Nos solos LVAd-1, LVAd-2 e LVd, o acréscimo dos valores de ITP com a elevação das doses de esterco bovino e de calcário significa maior liberação e aproveitamento do $\mathrm{P}$ nativo do solo e, ou, de fertilizantes adicionados aos mesmos $(<\mathrm{FCP})$. No solo RQo, que apresenta naturalmente baixa capacidade de retenção de $\mathrm{P}$, houve redução no processo de dessorção do nutriente, graças à contribuição da MO no processo de adsorção (Figura 1 e Quadros 2 e
3). A elevação da resistência de liberação de $\mathrm{P}$ da fração lábil (<ITP) no solo RQo, resultante da aplicação de matéria orgânica, pode significar menor perda do nutriente por lixiviação em solos de textura arenosa. Resultados semelhantes foram obtidos por Novak \& Watts (2004), os quais observaram efeito positivo da aplicação de resíduos de estação de tratamento de esgoto sobre o aumento da CMAP em solos arenosos e redução das perdas do nutriente por lixiviação. Como

Quadro 3. Estimativa e significância dos parâmetros dos modelos apresentados no quadro 2, e valores máximos de fósforo remanescente (P-rem) e do índice tampão de fósforo (ITP) e mínimo de capacidade máxima de adsorção de fósforo (CMAP) e respectivas quantidades ideais de esterco bovino (MO) e calcário (CAL), em cada solo

\begin{tabular}{lllcl}
\hline \multirow{3}{*}{ Parâmetro } & \multicolumn{3}{c}{ Solo } \\
\cline { 2 - 4 } & RQo & LVAd-1 & LVAd-2 & LVd \\
& & & & \\
\hline
\end{tabular}

\begin{tabular}{|c|c|c|c|c|}
\hline & \multicolumn{4}{|c|}{ P-rem } \\
\hline$\beta_{0}$ & $29,25( \pm 0,38) * * *$ & $29,81( \pm 0,39) * * *$ & $25,52( \pm 0,36) * * *$ & $12,22( \pm 0,29) * * *$ \\
\hline$\beta_{1}$ & $1,54( \pm 0,145) * * *$ & $1,36( \pm 0,15) * * *$ & $1,61( \pm 0,12) * * *$ & $0,62( \pm 0,04)^{* * *}$ \\
\hline$\beta_{2}$ & $2,69( \pm 0,29) * * *$ & $2,79( \pm 0,72) * * *$ & $3,11( \pm 0,60) * * *$ & $5,52( \pm 0,52)^{* * *}$ \\
\hline$\beta_{3}$ & $-0,05( \pm 0,02) * * *$ & $-0,06( \pm 0,01) * * *$ & $-0,08( \pm 0,01) * * *$ & $-2,08( \pm 0,23)^{* * *}$ \\
\hline$\beta_{4}$ & $-0,16( \pm 0,02) * * *$ & $-1,82( \pm 0,33) * * *$ & $-0,89( \pm 0,26) * * *$ & $-0,02( \pm 0,01)^{* * *}$ \\
\hline$\beta_{5}$ & - & - & $-0,26( \pm 0,04) * * *$ & - \\
\hline P-rem (máx.) & $40,70 \mathrm{mg} \mathrm{L}^{-1}$ & $38,48 \mathrm{mg} \mathrm{L}^{-1}$ & $33,69 \mathrm{mg} \mathrm{L}^{-1}$ & $21,82 \mathrm{mg} \mathrm{L}^{-1}$ \\
\hline \multirow[t]{2}{*}{ (MO; CAL) } & $(10,0 ; 0,61)$ & $(10,0 ; 0,77)$ & $(8,95 ; 0,42)$ & $(10,0 ; 1,27)$ \\
\hline & & & CMAP & \\
\hline$\beta_{0}$ & $307,32( \pm 6,76)^{* * *}$ & $402,49( \pm 10,39)^{* * *}$ & $781,08( \pm 13,1)^{* * *}$ & $1.266,98( \pm 13,34) * * *$ \\
\hline$\beta_{1}$ & $-29,44( \pm 2,12) * * *$ & $-67,69( \pm 7,52) * * *$ & $-28,09( \pm 4,37))^{* * *}$ & $-16,32( \pm 1,58) * * *$ \\
\hline$\beta_{2}$ & $-78,02( \pm 16,14) * * *$ & $-113,35( \pm 16,14) * * *$ & $-102,01( \pm 21,7) * * *$ & $-146,64( \pm 27,26) * * *$ \\
\hline$\beta_{3}$ & $1,29( \pm 0,18) * * *$ & $14,54( \pm 1,73) * * *$ & $0,790( \pm 0,39)^{*}$ & $66,19( \pm 12,64) * * *$ \\
\hline$\beta_{4}$ & $27,82( \pm 7,49) * * *$ & $47,71( \pm 6,66) * * *$ & $31,74( \pm 9,37)^{* *}$ & - \\
\hline$\beta_{5}$ & $9,45( \pm 2,64) * * *$ & $-0,87( \pm 0,11) * * *$ & $9,02( \pm 1,59)^{* * *}$ & - \\
\hline$\beta_{6}$ & $-3,4231( \pm 1,22) * *$ & $16,19( \pm 3,98) * * *$ & - & - \\
\hline$\beta_{7}$ & - & $-1,51( \pm 0,38) * * *$ & - & - \\
\hline CMAP(mín.) & $141,92 \mathrm{mg} \mathrm{kg}^{-1}$ & $254,59 \mathrm{mg} \mathrm{kg}^{-1}$ & $578,08 \mathrm{mg} \mathrm{kg}^{-1}$ & $1.022,57 \mathrm{mg} \mathrm{kg}^{-1}$ \\
\hline \multirow[t]{2}{*}{ (MO;CAL) } & $(10,0 ; 0,0)$ & $(10,0 ; 1,07)$ & $(10,0 ; 0,18)$ & $(10,0 ; 1,10)$ \\
\hline & & & ITP & \\
\hline$\beta_{0}$ & $3,86953( \pm 0,205)^{* * *}$ & $5,3207( \pm 0,1512)^{* * *}$ & $4,3367( \pm 0,0422)^{* * *}$ & $1,446 \times 10^{-2}\left( \pm 9,9 \times 10^{-4}\right)^{* * *}$ \\
\hline$\beta_{1}$ & $0,09886( \pm 0,0348) * *$ & $0,1142( \pm 0,0969) \mathrm{ns}$ & $-0,3399( \pm 0,032) * * *$ & $4,619 \times 10^{-3}\left( \pm 7,1 \times 10^{-4}\right)^{* * *}$ \\
\hline$\beta_{2}$ & $-0,26059( \pm 0,212) \mathrm{ns}$ & $-1,7273( \pm 0,354)^{* * *}$ & $-0,3551( \pm 0,07)^{* * *}$ & $-5,401 \times 10^{-4}\left( \pm 3,8 \times 10^{-3}\right) \mathrm{ns}$ \\
\hline$\beta_{3}$ & $2,41342( \pm 0,183)^{* * *}$ & $-0,0939( \pm 0,024)^{* * *}$ & $0,0471( \pm 0,0079)^{* * *}$ & $-7,456 \times 10^{-4}\left( \pm 1,6 \times 10^{-4}\right)^{* * *}$ \\
\hline$\beta_{4}$ & $-0,27077( \pm 0,025)^{* * *}$ & $0,5156( \pm 0,1642)^{* *}$ & $0,0962( \pm 0,033)^{* *}$ & $1,33 \times 10^{-2}\left( \pm 5,6 \times 10^{-3}\right)^{*}$ \\
\hline$\beta_{5}$ & $-0,92358( \pm 0,110)^{* * *}$ & $0,0068( \pm 0,1642)^{* * *}$ & $-0,002( \pm 0,0005)^{* * *}$ & $4,433 \times 10^{-5}\left( \pm 1,0 \times 10^{-5}\right)^{* * *}$ \\
\hline$\beta_{6}$ & $0,10548( \pm 0,0154)^{* *}$ & $0,2175( \pm 0,1642)^{* * *}$ & $0,042( \pm 0,007 * * *$ & $-5,533 \times 10^{-3}\left( \pm 1,9 \times 10^{-3}\right)^{* *}$ \\
\hline$\beta_{7}$ & - & $-0,0738( \pm 0,0268)^{* *}$ & $-0,0044( \pm 0,001)^{* * *}$ & $-1,43 \times 10^{-3}\left( \pm 3,7 \times 10^{-4}\right)^{* * *}$ \\
\hline$\beta_{8}$ & - & - & - & $9,127 \times 10^{-5}\left( \pm 3,6 \times 10^{-5}\right)^{* * *}$ \\
\hline ITP (máx.) & 0,126 & 0,0327 & 0,0391 & 0,0333 \\
\hline (MO;CAL) & $(10,0 ; 1,22)$ & $(7,5 ; 2,0)$ & $(10,0 ; 2,0)$ & $(10,0 ; 1,34)$ \\
\hline
\end{tabular}

$* * *, * *, *$,ns: significativo a 0,$1 ; 1$ e $5 \%$ e não significativo, respectivamente. 
o ITP representa a resistência do solo às mudanças do P-lábil, em conseqüência de alterações no $\mathrm{P}$ da solução, o manejo de Latossolos Argilosos que beneficie a elevação do $\mathrm{pH}$ e o incremento do teor de MO é essencial para a otimização do uso do P (Fox \& Kamprath, 1970).

O quadro 4 apresenta os coeficientes de correlação entre o P-rem, CMAP e ITP e a produção de grãos e o $P$ acumulado na parte aérea total (parte aérea + grãos) do feijoeiro, obtidos por Souza et al. (2006). Observase que houve correlações positivas e significativas entre o P-rem e ITP, tanto para a produção de grãos quanto para o $\mathrm{P}$ acumulado, em todos os solos, à exceção do ITP para o solo RQo; para a CMAP, as correlações foram significativas e negativas, à exceção do solo LVAd-1. Esses resultados mostram que a calagem e a adição de esterco bovino, como já discutido, reduziram a adsorção do $\mathrm{P}$ pelos solos, aumentando sua disponibilidade, possibilitando maior absorção do nutriente e maior produção de grãos pelo feijoeiro (Souza et al., 2006). Portanto, pode-se inferir que práticas de manejo que aumentem o teor de $\mathrm{MO}$ e o $\mathrm{pH}$ do solo são opções viáveis para a maximização da adubação fosfatada, principalmente, para pequenos agricultores que demonstram dificuldades para aquisição de fertilizantes minerais.

\section{CONCLUSÕES}

1. A adição de esterco bovino e de calcário incrementou os valores de P-rem e ITP e reduziu a CMAP, sendo as alterações nos solos, na seguinte ordem decrescente de intensidade: LVd > LVAd-2 > LVAd-1 > RQo.

2. As alterações nos valores de P-rem, CMAP e ITP foram condicionadas à mineralogia e textura, sendo a adsorção maior com o maior teor de óxidos dos solos. Quanto mais argiloso e intemperizado o solo, menores os valores observados de P-rem e ITP e maior a CMAP, conseqüentemente, menor a disponibilidade de $\mathrm{P}$.

\section{LITERATURA CITADA}

ACCIOLLY, L.J.O.; DALBÓ, M.A.; ALVAREZ V., V.H. \& RIBEIRO, A.C. Métodos para determinação do potencial de sulfato em solos. R. Bras. Ci. Solo, 9:103-106, 1985.

ALVAREZ V., V.H. Avaliação da fertilidade do solo (Superfícies de resposta - modelos aproximativos para expressar a relação fator-resposta). Viçosa, MG, Universidade Federal de Viçosa, 1994. 75p.

ALVAREZ V., V.H. \& FONSECA, D.M. Definição de doses de fósforo para determinação da capacidade máxima de adsorção de fosfatos e para ensaios de casa de vegetação. R. Bras. Ci. Solo, 14:48-55, 1990.

ALVAREZ V., V.H.; NOVAIS, R.F.; DIAS, L.E. \& OLIVEIRA, J.A. Determinação e uso do fósforo remanescente. B. Inf. Soc. Bras. Ci. Solo, 25:27-32, 2000.

BAHIA FILHO, A.F.C.; BRAGA, J.M.; RESENDE, M. \& RIBEIRO, A.C. Relação entre adsorção de fósforo e componentes mineralógicos da fração argila de Latossolos do Planalto Central. R. Bras. Ci. Solo, 7:221-226, 1983.

BRAGA, J.M. \& DEFELIPO, B.V. Determinação espectrofotométrica de fósforo em extratos de solo e material vegetal. R. Ceres, 21:73-85, 1974.

CURI, N. \& FRANZMEIER, D.P. Toposequence of Oxisols from the Central Plateau of Brazil. Soil Sci. Soc. Am. J., 48:341-346, 1984.

CURI, N.; CAMARGO, O.A.; GUEDES, A. \& SILVEIRA, J.V. Sorção de fósforo em materiais de Latossolos do Brasil sudeste. In: REUNIÃO DE CLASSIFICAÇÃO, CORRELAÇÃO DE SOLOS E INTERPRETAÇÃO DA APTIDÃO AGRÍCOLA, 3., Rio de Janeiro, 1988. Anais. Rio de Janeiro, Embrapa-SNLCS/SBCS, 1988. p.267-282.

DAY, P.R. Particle fractionation and particle-size analysis. In: BLACK, C.A. ed. Methods of soil analysis. Madison, America Society of America 1965. v.1. p.545-566.

Quadro 4. Coeficientes de correlação linear entre fósforo remanescente (P-rem), capacidade máxima de adsorção de $\mathbf{P}$ (CMAP) e índice tampão de fosfato (ITP) e produção de grãos e acúmulo de $\mathbf{P}$ (P-acum.) na parte aérea total (matéria seca da parte aérea + matéria seca de grãos) do feijoeiro

\begin{tabular}{|c|c|c|c|c|c|c|c|c|}
\hline & \multicolumn{8}{|c|}{ Solo } \\
\hline & \multicolumn{2}{|c|}{ RQo } & \multicolumn{2}{|c|}{ LVAd-1 } & \multicolumn{2}{|c|}{ LVAd-2 } & \multicolumn{2}{|c|}{ LVd } \\
\hline & Grãos & P-acum. & Grãos & P-acum. & Grãos & P-acum. & Grãos & P-acum. \\
\hline P-rem & $0,86^{* *}$ & $0,91 * *$ & $0,71^{*}$ & $0,69^{*}$ & $0,65^{*}$ & $0,87^{* *}$ & $0,78^{*}$ & $0,89^{* *}$ \\
\hline CMAP & $-0,85^{* *}$ & $-0,86 * *$ & $-0,07 \mathrm{~ns}$ & $-0,06 \mathrm{~ns}$ & $-0,58^{*}$ & $-0,84 * *$ & $-0,64^{*}$ & $-0,76^{*}$ \\
\hline ITP & $-0,40 \mathrm{~ns}$ & $-0,36 \mathrm{~ns}$ & $0,79 *$ & $0,71^{*}$ & $0,64^{*}$ & $0,78^{*}$ & $0,73^{*}$ & $0,75^{*}$ \\
\hline
\end{tabular}

\footnotetext{
*** e ns: significativos a 1 e 5\% e não-significativo, respectivamente, pelo teste de t.
} 
EMPRESA BRASILEIRA DE PESQUISA AGROPECUÁRIA EMBRAPA. Centro Nacional de Pesquisa de Solos. Sistema brasileiro de classificação de solos. Brasília, Embrapa Produção de informação; Rio de Janeiro, Embrapa Solos, 1999. 412p.

EMPRESA BRASILEIRA DE PESQUISA AGROPECUÁRIA EMBRAPA. Serviço Nacional de Levantamento e Conservação de Solo. Manual de métodos de análise de solo. Rio de Janeiro, Ministério da Agricultura, 1997. 212p.

FOX, R.L. \& KAMPRATH, E.J. Phosphate sorption isotherms for evaluating the phosphate requirements of soils. Soil Sci. Soc. Am. Proc., 34:902-907, 1970.

FROSSARD, E.; BROSSARD, M.; HEDLEY, M.J. \& METHERELL, A. Reactions controlling the cycling of $\mathrm{P}$ in soils. In: TIESSEN, H., ed. P cycling in terrestrial and aquatic ecosystem: a global perspective. New York, John Wiley \& Sons, 1994. p.1-65.

GUILHERME, L.R.G.; CURI, N.; SILVA, M.L.N.; BRENÓ, N.B. \& MACHADO, R.A.F. Adsorção de fósforo em solos de várzea do estado de Minas Gerais. R. Bras. Ci. Solo, 24:27 34,2000 .

HAYNES, R.J. Lime and phosphate in the soil-plant system. Adv. Agron., 37:249-315, 1984.

HUE, N.V. Effects of organic acids/anions on phosphorus sorption and phytoavailability in soils with different mineralogies. Soil Sci., 152:463-471, 1991.

IYAMUREMYE, F.; DICK, R.P. \& BAHAM, J. Organic amendments and phosphorus dynamics: Phosphorus chemistry and sorption. Soil Sci., 161:426-435, 1996.

KHURI, A.I. \& CORNELL, J.A. Response surfaces-designs and analyses. 2.ed. New York, 1996. 510p.

KLUG, H.P. \& ALEXANDER, L.E. X-ray diffraction procedures for polycrystalline and amorphous materials. New York, John Wiley, 1974. 716p.

KRISHNA MURTI, G.S.R.; VOLK, V.V. \& JACKSON, M.L. Calorimetric determination of iron of mixed valency by orthophenantroline. Soil Sci. Soc. Am. Proc., 30:663-664, 1966.

LOPES, A.S. \& COX, F.R. Relação de características físicas, químicas e mineralógicas com fixação de fósforo em solos sob cerrados. R. Bras. Ci. Solo, 3:82-88, 1979.

McBRIDE, M.B. Environmental chemistry of soils. New York, University Press, 1994. 406p.
MEHRA, O.P. \& JACKSON, N.L. Iron oxide removal from soils and clays by a dithionite-citrate system buffered with sodium bicarbonate. Clays and Clays Miner., 3:317-327, 1960.

NOVAIS, R.F. \& SMYTH, T.J. Fósforo em solo e planta em condições tropicais. Viçosa, MG, Universidade Federal de Viçosa, 1999. 399p.

NOVAK, J.M. \& WATTS, D.W. Increasing the phosphorus sorption capacity of south-eastern coastal plain soils using water treatment residuals. Soil Sci., 169:206-214, 2004.

OLSEN, S.R. \& WATANABE, F.S. A method to determine a phosphorus adsorption maximum of soils as measured by the Langmuir Isotherm. Soil Sci. Soc. Am. Proc., 21:144$149,1957$.

R DEVELOPMENT CORE TEAM. R: language and environment for statistical computing. R Foundation for Statistical computing, Vienna, Austria. 2004. Disponível em: <http://www.R-project.org>. acesso em: $25 \mathrm{dez} 2004$.

RAIJ, B. van. Fertilidade do solo e adubação. Piracicaba, Ceres, 1991. 343p.

RAIJ, B. van.; QUAGGIO, J.A.; CANTARELLA, H.; FERREIRA, M.E.; LOPES, A.S. \& BATAGLIA, O.C. Análise química do solo para fins de fertilidade. Campinas, Fundação Cargil, 1987. 170p.

RESENDE, M. Mineralogy, chemistry, morphology and geomorphology of some soils of the Central Plateau of Brazil. West Lafayette, Purdue University, 1976. 327p. (Tese de Doutorado)

RUAN, H.D. \& GILKES, R.J. Kinetics of phosphate sorption and desorption by synthetic aluminous goethite before and after thermal transformation to hematite. Clay Miner., 31:63-74, 1996.

SCHWERTMANN, U. Differenzierung der eisenoxide des bondes durch extraktion mit ammonium-oxalat-losung. Zeitschrift Pflanzernahrung, 105:194-202, 1964.

SOUZA, R.F.; FAQUIN, V.; FERNANDES, L.A. \& AVILA, F.W. Nutrição fosfatada e rendimento do feijoeiro sob influência da calagem e adubação orgânica. Ci. Agrotec., 30:656664, 2006. Disponível em: <http://www.editora.ufla.br/ revista/30_4/art10.pdf.> acesso em: 12 set 2006.

TORRENT, J.; BARRON, V. \& T.B. \& SCHWERTMANN, U. Phosphate adsortption and desorption by geothites differing in crystal morphology. Soil Sci. Soc. Am. J., 54:1007-1012, 1990 